\title{
Intermédialités
}

Histoire et théorie des arts, des lettres et des techniques

Intermediality

History and Theory of the Arts, Literature and Technologies

\section{« Mettre en scène »}

Une approche intermédiale de la réalité théâtrale actuelle

\section{George Brown, Gerd Hauck et Jean-Marc Larrue}

Numéro 12, automne 2008

Mettre en scène

Directing

URI : https://id.erudit.org/iderudit/039228ar

DOI : https://doi.org/10.7202/039228ar

Aller au sommaire du numéro

Éditeur(s)

Centre de recherche sur l'intermédialité

ISSN

1705-8546 (imprimé)

1920-3136 (numérique)

Découvrir la revue

Citer ce document

Brown, G., Hauck, G. \& Larrue, J.-M. (2008). « Mettre en scène " : une approche intermédiale de la réalité théâtrale actuelle. Intermédialités / Intermediality,

(12), 9-12. https://doi.org/10.7202/039228ar d'utilisation que vous pouvez consulter en ligne. 


\title{
«Mettre en scène »
}

\author{
Une approche intermédiale \\ de la réalité théâtrale actuelle
}

\author{
George Brown \\ GERD HaUCK \\ Jean-Marc larrue
}

e numéro d'Intermédialités marque un nouveau pas dans la pénétration de l'approche intermédiale dans le champ des études théâtrales. Bien qu'on relève l'influence grandissante de la pensée intermédiale chez des chercheurs et théoriciens du théâtre au cours des quinze dernières années, on note une réticence du monde du théâtre à adopter cette autre et nouvelle façon de percevoir et de concevoir sa pratique. Ce n'est qu'en 2006 que l'intermédialité fait une première incursion majeure et s'affiche dans ce terrain a priori peu hospitalier grâce à l'ouvrage Intermediality in Theatre and Performancel, publié sous la direction de Freda Chapple et Chiel Kattenbelt. La série d'essais que contient ce numéro prend comme point focal la mise en scène. Pourquoi ce choix? D'une part, parce que l'apparition de la mise en scène est contemporaine de la révolution électrique et, d'autre part, parce qu'elle subit au premier chef les pressions intermédiatiques et celles des nouvelles technologies. C'est également la mise en scène qui compose avec l'acteur, module sa présence - corporelle, visuelle, sonore - et celle du public.

Lapproche intermédiale change radicalement les perspectives et remet en cause des hypothèses qui, au fil des décennies, s'étaient transformées en vérités immuables fondant l'essence même de la pratique. On ne s'étonne donc pas des résistances qu'elle suscite et suscitera encore. Mais en même temps, rares sont ceux au sein des études théâtrales qui ne reconnaissent pas la nécessité et l'urgence de revoir l'histoire du théâtre, de sa dynamique et, surtout, de ses

1. Freda Chapple et Chiel Kattenbelt (dir.), Intermediality in Theatre and Performance, Amsterdam et New York, Rodopi, 2006. 
rapports complexes et nombreux avec les autres médias, ce qui inclut, dans une perspective intermédiale, les autres pratiques artistiques.

Ce numéro s'ouvre donc sur un article de Jean-Marc Larrue qui explore les différentes causes pouvant expliquer cette rencontre tardive - si on la compare avec ce qui s'est produit dans d'autres pratiques - du théâtre et de l'intermédialité. On ne sera pas surpris de l'importance du concept de "présence », qui se développe sur près d'un siècle, dans ce phénomène de « résistance ». On ne s'étonnera pas non plus que sur six des articles suivants, trois portent spécifiquement sur le son. Parent pauvre des études théâtrales traditionnelles, oublié par les historiens, le son est pourtant l'une des composantes essentielles de la pratique. Si le théâtre est un art de la vue - où l'on voit, où l'on est vu —, il est aussi un art du son, celui de la scène, bien sûr, mais aussi celui du public et celui du lieu où se produit la représentation. Il est normal que, dans une perspective intermédiale, qui se fonde sur les dynamiques des relations, le son occupe une position centrale, à la mesure de celle qu'il occupe dans la réalité de la pratique.

Après avoir abordé la question des possibles «causes du retard », ce dossier consacré à la mise en scène s'ouvre par une importante mise en garde. Certains créateurs, Bertolt Brecht en tête, ont lié l'utilisation sur scène des nouvelles technologies ou d'autres médias à des projets de transformation radicale du théâtre ou de la société. Il découle de cette pratique, et des travaux qui lui ont été consacrés, une équation en apparence anodine qui lie, au théâtre, radicalisme et nouvelles technologies. C'est sur cet amalgame que se penche Robin Nelson, dans «After Brecht: the Impact (Effects, Affects) of Intermedial Theatre ». Cet essai s'articule autour de deux questions essentielles:

1. «[D]oes intermedial theatre dispose some impacts/effects/affects rather than others? » and, taking up the references to Brecht,

2. «Does intermedial theatre have an inherently radical disposition and, if so, what kind of radicalism²?»

Après le réexamen rigoureux et solidement étayé de Nelson, Tatiana Burtin propose une exploration du concept d'interartialité tel que l'a développé Walter Moser. Prenant pour base de sa réflexion les spectacles «Art» de Yasmina Reza et Seuls de Wajdi Mouawad, Burtin explore les défis que pose la théâtralisation de la peinture, soit dans le dialogue, soit dans l'action dramatique, ce qui ramène à l'acteur et à sa fonction médiatrice. "Questions de cadres: mise en scène de la peinture dans «Art» de Yasmina Reza et Seuls de Wajdi Mouawad» 
élargit et renouvelle la réflexion sur l'interartialité comme modalité spécifique de l'intermédialité.

Cette première section se termine par l'examen d'une pratique singulière, le progetto, qu'Erica Magris assimile à un «projet multithéâtral ». Développé en Italie par la "Génération 90 », le progetto est conçu comme un parcours de quelques années autour d'un thème, d'une œuvre ou d'un auteur. Les créateurs avancent par étapes, "en réalisant non seulement des spectacles ou des performances, mais en se consacrant également à d'autres formats artistiques et en faisant usage de plusieurs médias" ». Cet essai intitulé "Le "projet multithéâtral" - Transformations intermédiales des scènes italiennes contemporaines» offre une excellente illustration de la dynamique intermédiale qui lie le théâtre aux autres pratiques.

La deuxième section de ce numéro regroupe, tel qu'indiqué plus tôt, trois études consacrées au son qui abordent le phénomène de l'audition et de la production sonore selon trois perspectives différentes. Partant de certaines observations faites au sujet de spectacles de Marie Brassard, dont Jimmy, créature de rêve, Michael Darroch examine la relation de la voix humaine avec l'espace théâtral lors de la représentation. "Digital Multivocality and Embodied Language in Theatrical Space » soulève la question de la matérialité de la voix humaine sous l'effet des technologies numériques, ce qui conduit l'auteur à s'interroger sur la nature même de la médialité du théâtre actuel. «[T] oday’s voice can not only be recorded, cut up and spliced together again, but also modulated, reconfigured or simply generated via modern speech technologies ${ }^{4}$. » Plutôt que de comprendre la voix humaine comme l'expression de l'auteur, Darroch démontre que les technologies numériques de reproduction sonore ont plutôt révélé son statut de média, avec pour résultat, comme c'est le cas chez Marie Brassard, un recentrage de l'espace théâtral sur la voix humaine, devenue le cœur d'une expérience synesthésique.

Approfondissant dans ces pages une réflexion menée depuis quelques années sur le son au théâtre, Marie-Madeleine Mervant-Roux analyse une tendance nouvelle qui se dessine sur la scène contemporaine et qui entraîne un renouvellement palpable de la réception chez le spectateur. "Quand le son écoute la scène. Une exploration inédite de la matière théâtrale» scrute les causes possibles de ce changement. Selon Mervant-Roux, "c'est l'articulation de deux médias, le décalage volontairement maintenu entre deux systèmes techniques et symboliques distincts, un médi[a] archaïque, le théâtre, et un médi[a] à l'état naissant, 
l'écriture sonore, qui provoqu[e] chez le spectateur un jeu entre un mode non théâtral d'écoute et le traditionnel mélange de vision et d'audition, entraînant finalement un renouvellement du regard ${ }^{5}$ ».

Il était normal que ce numéro qui s'ouvre par une discussion sur le rôle central de l'acteur dans l'épistémè et la réalité du théâtre consacre ses dernières pages à ce même acteur. «Sense \& Sensation: the act of mediation and its effects » rend compte d'une expérience audacieuse et rigoureuse par laquelle les technologies numériques démultiplient et transforment les capacités d'expression sonore du corps. Julie Wilson-Bokowiec et Mark Bokowiec y montrent comment, contrairement à ce que suggère le discours essentialiste, le mariage du corps de l'acteur et des technologies enrichit l'expérience théâtrale. Ce corps «augmenté» par des capteurs hypersensibles, qui transforment le geste en son et qui modulent la voix, crée un univers sonore et visuel inexploré qui n'est pas sans évoquer la «voix suicidée» d'Artaud et le rêve de cette voix profonde que la tradition théâtrale a rendue inaudible.

«Mettre en scène » à l'ère intermédiale implique non seulement un renouvellement des pratiques mais aussi une redéfinition des relations entre les créateurs. C'est ce qu'illustre le dossier visuel que Johanne Lamoureux a constitué autour du travail scénographique que le sculpteur Michel Goulet a réalisé, depuis près de 20 ans, dans le cadre de ses collaborations avec le metteur en scène Denis Marleau et le Théâtre Ubu.

Ce numéro se termine par un texte inédit de Paul Zumthor (1915-1995) retrouvé et présenté par Philippe Despoix. Médiéviste réputé, partisan d'une approche comparatiste généralisée des phénomènes culturels et historiques, Paul Zumthor a été l'un des principaux promoteurs du concept de poésie orale ${ }^{6}$. Dans ce texte, l'un des ses derniers (rédigé entre 1989 et 1994), l'auteur poursuit sa réflexion sur l'oralité, qu'elle soit médiatisée ou non, en examinant particulièrement sa dimension performative : la valeur qualitative de la vocalité, l'importance du corps et du geste comme médium de la voix, le lien indissociable entre parole poétique et chant. L'intérêt de ce texte réside autant dans la richesse et l'originalité des concepts qui y sont développés que dans son approche de l'oralité que Zumthor perçoit comme une forme élémentaire de théâtralité.

5. Supra., p. 117.

6. C'est le titre d'un de ses ouvrages marquants: Introduction à la poésie orale, Paris, Éditions du Seuil, coll. «Poétique », 1983. 